\title{
Recomendación sobre Curvas de Crecimiento Intrauterino
}

\author{
MARCELA MILAD A. ${ }^{1,2}$, JOSÉ M. NOVOA P. ${ }^{1,3}$, JORGE FABRES B. ${ }^{4}$, \\ $M^{\text {a }}$ MARGARITA SAMAMÉ M. ${ }^{5}$, CARLOS ASPILLAGA M., \\ Directiva Rama de Neonatología, Sociedad Chilena de Pediatría \\ 1. Director Rama de Neonatología. \\ 2. Jefe de Neonatología Clínica Santa María. Docente Departamento de Pediatría, Universidad de Los Andes. \\ 3. Jefe de Neonatología Hospital Padre Hurtado. Profesor Departamento de Pediatría, Facultad de Medicina Universidad del \\ Desarrollo Clínica Alemana. \\ 4. Presidente Rama de Neonatología. Profesor Asistente de Pediatría, Departamento de Pediatría, Pontificia Universidad \\ Católica de Chile. Servicio de Neonatología Hospital Clínico Pontificia Universidad Católica de Chile. \\ 5. Vice-Presidente Rama de Neonatología, Profesor Adjunto, Departamento de Pediatría, Universidad de Chile Campus \\ Occidente. Servicio de Neonatología Hospital San Juan de Dios. \\ 6. Profesor Asistente de Pediatría, Departamento de Obstetricia y Ginecología. Universidad de Chile Campus Centro. \\ Miembro del Consejo de la Escuela de Medicina Universidad de Chile.
}

Se considera que el monitoreo del crecimiento intrauterino constituye una parte importante de la evaluación de salud y nutrición del neonato al nacer y adquiere especial importancia en el RN de pretérmino, ya que en este grupo, el peso y la edad gestacional, son criterios básicos para definir poblaciones de mayor o menor riesgo de mortalidad y morbilidad neonatal ${ }^{1-3}$. De la definición adecuada de este grupo de riesgo, se implementan intervenciones obstétricas y neonatales destinadas a aminorar los riesgos y evitar complicaciones a corto y largo plazo.

Existe una relación inversa entre el percentil de peso fetal y resultado adverso perinatal, estando el riesgo mayor bajo el percentil 3 para la edad gestacional ${ }^{4,5}$. Por otro lado, la restricción del crecimiento intrauterino (RCIU) pareciera ser un importante determinante de riesgo para hipertensión arterial, hiperlipidemia, enfermedad cardiaca coronaria y diabetes mellitus en la vida adulta ${ }^{6,7}$.
En el análisis del tipo de curva de crecimiento óptimo, se describen principalmente 2 tipos $^{8}$; curvas de crecimiento estándar (población seleccionada) que representa un ideal de crecimiento sano de una población y curvas de crecimiento referencial (poblacional) en la cual se describe una población, sin ninguna referencia al estado de salud de la misma. De lo anteriormente señalado, las primeras cumplen el objetivo de compararse con un ideal de crecimiento y por lo tanto tener un mayor nivel de sensibilidad y especificidad en sus poblaciones extremas.

La Organización Mundial de la Salud recomienda la realización de curvas de crecimiento intrauterino en lo posible en cada centro perinatológico o en cada país, con el objetivo que sean representativos de esa población y ha establecido además, criterios de validación de ellas $^{9,10}$.

La Rama de Neonatología de la Sociedad Chilena de Pediatría, ha realizado una revisión

Trabajo recibido el 17 de agosto de 2009, aceptado para publicación el 18 de enero de 2010.

Correspondencia a:

Marcela Milad A.

E-mail:mmilad@csm.cl 
de las últimas Curvas de Crecimiento Intrauterino publicadas en Chile, con el objetivo de determinar una recomendación sobre aquella curva de peso, talla y circunferencia de cráneo, que tenga una mayor representatividad de la población chilena y que permita diagnosticar con mayor sensibilidad y especificidad la población en riesgo, especialmente la Restricción de Crecimiento Intrauterino (RCIU), sobre la cual intervenir en forma precoz evitando secuelas a corto y largo plazo.

\section{Revisión de Curvas de Crecimiento Intrauterina Publicadas}

Se revisan las curvas de crecimiento, publicadas en la literatura nacional en los últimos 25 años. Se analiza sus similitudes y diferencias y la correlación con morbimortalidad neonatal.

Para este fin se analizan 4 curvas de crecimiento de niños chilenos; curva publicada por Juez y cols en el año 1984, Pittaluga y cols en el año 2002, González y cols en el año 2004 y Alarcón en el año 2008.

La revisión de las diferentes curvas nacionales revela:

\section{Curvas Juez:}

Publicada en el año 1984, es un estudio prospectivo de una cohorte de $11543 \mathrm{RN}$, nacidos en la maternidad de la Pontificia Universidad Católica de Chile, entre las 26 y 42 semanas de edad gestacional entre los años 1978 y 1984. Esta curva está constituida por RN nacidos vivos únicos, hijos de madres sanas, sin factores de riesgo perinatal y sin malformaciones congénitas ${ }^{11}$.

\section{Curvas Pittaluga:}

Publicada en el año 2002. Curvas de crecimiento intrauterino para prematuros entre 23 a 36 semanas de edad gestaciona ${ }^{16}$. Este estudio se realizó en base a datos recopilados prospectivamente entre los años 1990 a 1998, en el Hospital Sótero del Río, Región Metropolitana, Santiago. Se incluyeron $2830 \mathrm{RN}$ menores de 37 semanas, con edad gestacional confiable, producto de embarazos únicos y sin patología materna y fetal ${ }^{12}$.

\section{Curvas González:}

Publicada en el año 2004. Es una curva poblacional, referencial, con un n de 2049446 sujetos entre las edades 24 y 42 semanas. Se incluyen todos los $\mathrm{RN}$ únicos, vivos, nacidos en Chile entre los años 1993 y 2000, con datos obtenidos del Instituto Nacional de Estadísticas (INE) y del Departamento de Informática del Ministerio de Salud de Chile, sin discriminación de patología materna, placentaria o fetal ${ }^{13}$.

\section{Curvas Alarcón:}

Publicada en el año 2008. Se analiza una población de 148395 neonatos, del Servicio de Salud Metropolitano Oriente, de las maternidades del Hospital Salvador entre los años 1988-2000 y Hospital Luis Tisné entre los años 2001-2005. Se trata de una población seleccionada de $\mathrm{RN}$, entre las edades gestacionales 24 y 42 semanas, con determinación de edad gestacional confiable y excluyendo a aquellos hijos de madres con patología asociada ${ }^{14}$.

\section{Análisis}

Se evalúan estas 4 curvas de crecimiento, 3 de población seleccionada y una curva de crecimiento poblacional en la detección de morbilidad y mortalidad neonatal.

Carvajal y $\operatorname{cols}^{15}$, comparan la morbilidad, mortalidad y antropometría de RN con restricción de crecimiento y con crecimiento normal en un mismo período, entre las curvas de Juez y González. Se analiza una muestra de 11586 RN entre los años 2000 a 2004, comparando 3 grupos, grupo A, niños bajo el percentil 10 para Juez pero adecuados para González; grupo B bajo el percentil 10 para González y Grupo $\mathrm{C}$ un grupo control entre el percentil 25 y 75 para Juez, pareados por edad gestacional (EG) y sexo con el grupo estudio A.

El estudio demuestra que de la población estudiada se cataloga un $9 \%$ con RCIU por curva de Juez versus un 4,3\% por la curva de González y concomitante con esto, la morbilidad neonatal característica de RCIU, poliglobulia e hipoglicemia, se encuentran subdiagnosticadas por González. No se demuestra efecto sobre mortalidad.

Se revisa además, estudio presentado por 
Pittaluga ${ }^{16}$, cuyo objetivo fue comparar el estándar de peso neonatal de la curva basada en datos poblacionales (curva González) versus curva de población seleccionada (curva Pittaluga-Juez) y su correlación con morbimortalidad. Se analizan 38795 partos consecutivos realizados en el quinquenio 2001 a 2005, abarcando edades gestacionales entre 22 y 42 semanas, seleccionado por población de madres sanas.

Se presentan tres grupos de adecuación: PEG común, clasificados como PEG por ambas curvas de referencia, AEG común, clasificados como adecuados por ambas curvas y el grupo Delta PEG, clasificados como PEG según curva Juez- Pittaluga y AEG para curva González.

Se realizó comparación de riesgo por modelo de regresión logística entre los PEG diagnosticados por curva Juez-Pittaluga y no diagnosticados por curva González (grupo Delta PEG) versus los AEG comunes a ambas curvas lo cual se expresa en la tabla 1 extraída de lo presentado por Pittaluga ${ }^{16}$.

La comparación de ambas curvas, poblacional (González) y seleccionada (Juez-Pittaluga), en relación a los riesgos de mortinatalidad y morbilidad, claramente establece que la sensibilidad de la detección es mayor en la

Tabla 1. Grupo PEG no diagnosticado por curva poblacional versus AEG común a ambas curvas

\begin{tabular}{|lccc|}
\hline Patología & OR & IC 95\% & p \\
\hline Mortinato & 3,26 & $2,12-5,01$ & $\mathrm{p}<0,0001$ \\
\hline Fallecer & 1,64 & $0,99-2,72$ & $\mathrm{p} 0,054$ NS \\
\hline Hospitalización & 2,24 & $2,01-2,49$ & $\mathrm{p}<0,0001$ \\
\hline Apgar 1' $\leq$ a 3 & 1,93 & $1,57-2,37$ & $\mathrm{p}<0,0001$ \\
\hline Apgar 5' $\leq$ a 6 & 2,10 & $1,56-2,82$ & $\mathrm{p}<0,0001$ \\
\hline Malformaciones & 1,75 & $1,33-2,31$ & $\mathrm{p}<0,0001$ \\
\hline Hipoglicemia & 5,52 & $4,19-7,27$ & $\mathrm{p}<0,0001$ \\
\hline Poliglobulia & 6,46 & $4,48-9,32$ & $\mathrm{p}<0,0001$ \\
\hline HIC severa & 3,34 & $1,67-6,68$ & $\mathrm{p}<0,001$ \\
\hline DBP & 3,29 & $2,34-4,63$ & $\mathrm{P}<0,0001$ \\
\hline
\end{tabular}

Modificado de Pittaluga ${ }^{16}$. curva con población seleccionada, siendo estadísticamente significativa en todas las patologías evaluadas. Mortalidad neonatal presenta tendencia no alcanzando significación estadística, pero si el riesgo de mortinato con OR de 3,26 у $\mathrm{p}<0,0001$.

Posteriormente en el año 2008, Alarcón ${ }^{14}$ publica curvas de crecimiento en población seleccionada y realiza un estudio comparativo con las otras curvas analizadas, incluye Juez, González y Pittaluga. Compara los promedios y DS de las diferentes curvas. Los promedios no son significativamente diferentes con las curvas de Juez y Pittaluga, pero significativamente superiores con la curva poblacional de González.

Alarcón realiza además un detallado análisis comparativo del p10 de las diferentes curvas, demostrando diferencias significativas en este percentil en los rangos 24 a 35 sem y 36 a 42 semanas con las curvas de González y similitud en el rango 24 a 35 sem con Pittaluga y 36 a 42 con Juez.

La Organización Mundial de la Salud (OMS) definió los criterios para que una curva de referencia sea considerada como un estándar apropiado; esto es, que sean realizadas en base a grandes poblaciones, que tengan al menos 200 niños en cada tramo de edad gestacional, deben incluir más de una variable antropométrica y con procedimientos muestrales definidos y reproducibles, entre otros factores. Estas curvas, si son tomadas de la población general, describen distribución poblacional y no necesariamente un patrón de normalidad. Esta situación se hace especialmente muy evidente, en prematuros menores de 33 semanas, dado que estos nacimientos están altamente influenciados por patologías maternas y/o placentarias que determinan disminución del peso de nacimiento y muchas veces son la causa del parto prematuro.

Una prioridad significativa, es además utilizar curvas actualizadas ya que los patrones de alimentación y cuidados de la madre se han modificado a través del tiempo y por lo tanto también el potencial de crecimiento del niño ${ }^{23-28}$.

Otro aspecto a considerar en las curvas de crecimiento, es el peso específico de los factores étnicos, de aquí la importancia de la 
existencia de curvas locales. Sin embargo, se plantea también que la variación étnica pudiera estar relacionada a factores como nutrición materna, salud y adecuados cuidados perinatales.

Uno de los principales objetivos de las curvas de crecimiento, es determinar la población en riesgo de enfermar o morir, por lo que es importante contar con un patrón de crecimiento lo más fisiológico posible y que permita el máximo de confiabilidad, con alta sensibilidad y especificidad. La detección adecuada de este grupo de fetos y/o RN permitirá la implementación oportuna de controles y/o medidas para disminuir los riesgos y para poder tratar a los casos en que la situación lo amerite.

Del análisis de las curvas de crecimiento intrauterino presentadas, la curva poblacional de González cumple con los requisitos de un número significativo en cada tramo de edad gestacional, pero subdiagnostica un grupo significativo de niños con RCIU. Es evidente que las curvas seleccionadas de población (Juez, Pittaluga y Alarcón), tienen una mejor sensibilidad y especificidad en la detección de morbilidad y mortalidad lo que queda demostrado en los estudios analizados.

Considerando que el tamaño muestral es una de las importantes limitaciones que tienen las curvas de población seleccionada, y que en los estudios realizados existe similitudes y concordancia temporal entre las curvas Alarcón y Pittaluga, se plantea la integración de estas curvas; Pittaluga en los tramos de peso 24 a 36 semanas, sumado a los datos obtenidos por Alarcón de las 24 a 42 semanas. La suma de la información de ambos autores, permite acercarse a lo sugerido por la OMS como óptimo de número necesario por cada tramo de edad gestacional.

Se demuestra absoluta coincidencia y similitud en promedios y DS de los diferentes tramos de EG. La sumatoria de estas curvas, para este fin denominadas como Curvas AlarcónPittaluga, permite la validación de una curva única de referencia que cumple con importantes requisitos de confiabilidad a saber, datos actualizados de población chilena, número significativo en cada tramo de EG y adecuada sensibilidad en la pesquisa de población de riesgo de morbilidad y mortalidad infantil.

La publicación original de Pittaluga analiza el peso por EG, por lo cual se la contactó para poder disponer de la información de talla de la misma población, datos que estaban registrados en la matriz y permitieron optimizar el $n^{\circ}$ de sujetos con talla para cada semana de edad gestacional.

La información aportada por Pittaluga y cols, corresponde al $28,6 \%$ de los datos de peso y talla en neonatos de 24 a 36 semanas de EG; todo el resto de la información corresponde a los datos de Alarcón y colaboradores. El $\mathrm{n}^{\mathrm{o}}$ de casos con información analizada es $91562 \mathrm{RN}$ en peso, 88445 casos en talla y 82604 neonatos en circunferencia craneana.

Las tablas 2-3-4 y 5 detallan información conjunta de peso, talla, circunferencia craneana e índice ponderal, respectivamente, con promedios, desviación estándar y percentiles ajustados de recién nacidos según edad gestacional. Además se grafica: figura 1 la curva de peso, figura 2 la curva de talla y perímetro craneano y en figura 3 el índice ponderal.

\section{Recomendación}

Por lo anteriormente expuesto, la Recomendación de la Rama de Neonatología de la Sociedad Chilena de Pediatría es utilizar las curvas antropométricas de población seleccionada que permiten pesquisar recién nacidos con crecimiento intrauterino insuficiente los cuales presentan significativo mayor riesgo en salud.

Para este fin, la información generada por los datos integrados de Alarcón y Pittaluga nos permitiría usar curvas representativas de la población chilena, actualizadas y con una mejor sensibilidad y especificidad en la detección de la población de riesgo.

La Rama de Neonatología de la Sociedad Chilena de Pediatría espera que estas Curvas de Crecimiento Intrauterino con información agregada de las Curvas presentadas por el Dr. Alarcón y de la Dra. Pittaluga vengan a complementar la información necesaria para pesquisar población de riesgo y permita enfrentar adecuadamente las patologías asociadas. 
Tabla 2. Peso; promedio, desviación estándar y percentiles ajustados de RN

\begin{tabular}{|c|c|c|c|c|c|c|c|c|c|}
\hline EG. Sem. & $\mathbf{n}$ & Promedio (g) & DS & p 3 & p 10 & p 25 & p 50 & p 75 & p 90 \\
\hline 24 & 85 & 766,3 & 102,8 & 601,0 & 640,6 & 691,0 & 749,1 & 835,0 & 897,9 \\
\hline 25 & 70 & 816,1 & 119,5 & 613,5 & 666,0 & 733,8 & 808,7 & 894,1 & 963,3 \\
\hline 26 & 106 & 904,0 & 138,5 & 660,9 & 728,2 & 812,4 & 903,5 & 992,6 & 1070,6 \\
\hline 27 & 99 & 1025,3 & 159,3 & 739,4 & 822,9 & 922,6 & 1029,2 & 1125,9 & 1214,6 \\
\hline 28 & 136 & 1175,4 & 181,6 & 845,0 & 945,7 & 1060,0 & 1181,4 & 1288,9 & 1390,1 \\
\hline 29 & 136 & 1349,6 & 204,9 & 973,8 & 1092,2 & 1220,3 & 1355,8 & 1476,9 & 1592,0 \\
\hline 30 & 180 & 1543,3 & 228,8 & 1122,0 & 1258,2 & 1399,1 & 1548,2 & 1685,0 & 1815,0 \\
\hline 31 & 219 & 1751,9 & 253,0 & 1285,6 & 1439,2 & 1592,0 & 1754,3 & 1908,3 & 2053,8 \\
\hline 32 & 317 & 1970,7 & 276,9 & 1460,8 & 1630,8 & 1794,8 & 1969,7 & 2141,9 & 2303,4 \\
\hline 33 & 352 & 2195,1 & 300,3 & 1643,6 & 1828,7 & 2003,0 & 2190,2 & 2380,9 & 2558,5 \\
\hline 34 & 656 & 2420,4 & 322,6 & 1830,2 & 2028,6 & 2212,3 & 2411,4 & 2620,5 & 2813,9 \\
\hline 35 & 1166 & 2642,0 & 343,6 & 2016,6 & 2226,0 & 2418,4 & 2629,1 & 2855,9 & 3064,4 \\
\hline 36 & 3079 & 2855,2 & 362,7 & 2198,9 & 2416,7 & 2617,0 & 2839,0 & 3082,1 & 3304,7 \\
\hline 37 & 6738 & 3055,4 & 379,6 & 2373,4 & 2596,2 & 2803,6 & 3036,7 & 3294,2 & 3529,8 \\
\hline 38 & 17974 & 3238,0 & 393,8 & 2536,0 & 2760,2 & 2973,9 & 3218,0 & 3487,5 & 3734,4 \\
\hline 39 & 26752 & 3398,3 & 405,0 & 2682,8 & 2904,2 & 3123,7 & 3378,5 & 3657,0 & 3913,2 \\
\hline 40 & 22339 & 3531,6 & 412,8 & 2810,0 & 3024,1 & 3248,4 & 3514,1 & 3797,9 & 4061,2 \\
\hline 41 & 10237 & 3633,4 & 416,7 & 2913,7 & 3115,3 & 3343,9 & 3620,2 & 3905,3 & 4173,0 \\
\hline 42 & 921 & 3698,9 & 416,4 & 2989,9 & 3173,5 & 3405,7 & 3692,8 & 3974,3 & 4243,5 \\
\hline Total & 91562 & & & & & & & & \\
\hline
\end{tabular}

Información conjunta Alarcón y Pittaluga.

Tabla 3. Talla; promedio, desviación estándar y percentiles ajustados de RN

\begin{tabular}{|c|c|c|c|c|c|c|}
\hline EG. Sem & $\mathbf{n}$ & Promedio (cm) & DS & p10 & p50 & p 90 \\
\hline 24 & 81 & 32,0 & 2,5 & 29,8 & 31,5 & 35,0 \\
\hline 25 & 68 & 33,3 & 2,6 & 30,9 & 32,8 & 36,2 \\
\hline 26 & 104 & 34,7 & 2,6 & 32,1 & 34,2 & 37,5 \\
\hline 27 & 95 & 36,0 & 2,6 & 33,4 & 35,6 & 38,8 \\
\hline 28 & 134 & 37,5 & 2,6 & 34,8 & 37,0 & 40,2 \\
\hline 29 & 135 & 38,9 & 2,5 & 36,2 & 38,4 & 41,5 \\
\hline 30 & 180 & 40,3 & 2,4 & 37,6 & 39,9 & 42,9 \\
\hline 31 & 218 & 41,7 & 2,3 & 39,0 & 41,3 & 44,2 \\
\hline 32 & 316 & 43,0 & 2,2 & 40,4 & 42,7 & 45,5 \\
\hline 33 & 352 & 44,3 & 2,1 & 41,7 & 44,0 & 46,8 \\
\hline 34 & 655 & 45,6 & 2,0 & 43,0 & 45,3 & 48,0 \\
\hline 35 & 1165 & 46,7 & 1,8 & 44,2 & 46,4 & 49,1 \\
\hline 36 & 2991 & 47,8 & 1,7 & 45,4 & 47,5 & 50,1 \\
\hline 37 & 6481 & 48,7 & 1,6 & 46,3 & 48,5 & 51,0 \\
\hline 38 & 17243 & 49,5 & 1,5 & 47,2 & 49,3 & 51,7 \\
\hline 39 & 25793 & 50,2 & 1,5 & 47,9 & 49,9 & 52,4 \\
\hline 40 & 21562 & 50,8 & 1,4 & 48,4 & 50,4 & 52,8 \\
\hline 41 & 9956 & 51,1 & 1,4 & 48,7 & 50,7 & 53,1 \\
\hline 42 & 916 & 51,3 & 1,4 & 48,8 & 50,8 & 53,2 \\
\hline Total & 88445 & & & & & \\
\hline
\end{tabular}

Información conjunta Alarcón y Pittaluga. 
RECOMENDACIÓN SOBRE CURVAS DE CRECIMIENTO INTRAUTERINO

Tabla 4. Perímetro cefálico; promedio, desviación estándar y percentiles ajustados de RN

\begin{tabular}{|c|c|c|c|c|c|c|}
\hline EG. Sem & $\mathbf{n}$ & Promedio $(\mathrm{cm})$ & DS & p10 & p50 & p 90 \\
\hline 24 & 6 & 23,0 & 1,0 & 21,9 & 23,2 & 24,4 \\
\hline 25 & 13 & 24,1 & 1,2 & 22,8 & 24,3 & 25,7 \\
\hline 26 & 13 & 25,1 & 1,3 & 23,6 & 25,3 & 26,9 \\
\hline 27 & 16 & 26,2 & 1,4 & 24,5 & 26,4 & 28,1 \\
\hline 28 & 26 & 27,2 & 1,5 & 25,5 & 27,4 & 29,1 \\
\hline 29 & 23 & 28,1 & 1,5 & 26,4 & 28,3 & 30,1 \\
\hline 30 & 37 & 29,1 & 1,5 & 27,3 & 29,3 & 31,0 \\
\hline 31 & 68 & 30,0 & 1,5 & 28,1 & 30,2 & 31,8 \\
\hline 32 & 143 & 30,8 & 1,5 & 29,0 & 31,0 & 32,6 \\
\hline 33 & 226 & 31,6 & 1,4 & 29,8 & 31,8 & 33,3 \\
\hline 34 & 412 & 32,3 & 1,4 & 30,5 & 32,5 & 33,9 \\
\hline 35 & 799 & 33,0 & 1,3 & 31,2 & 33,1 & 34,5 \\
\hline 36 & 2128 & 33,6 & 1,3 & 31,9 & 33,7 & 35,0 \\
\hline 37 & 6193 & 34,1 & 1,2 & 32,4 & 34,2 & 35,5 \\
\hline 38 & 16458 & 34,5 & 1,2 & 32,9 & 34,6 & 35,9 \\
\hline 39 & 24752 & 34,9 & 1,2 & 33,2 & 34,9 & 36,2 \\
\hline 40 & 20760 & 35,1 & 1,3 & 33,4 & 35,1 & 36,5 \\
\hline 41 & 9625 & 35,2 & 1,4 & 33,6 & 35,2 & 36,8 \\
\hline 42 & 906 & 35,3 & 1,5 & 33,5 & 35,2 & 37,0 \\
\hline Total & 82604 & & & & & \\
\hline
\end{tabular}

Información conjunta Alarcón y Pittaluga.

Tabla 5. Índice ponderal. Percentiles

\begin{tabular}{|cccc|}
\hline EG. Sem & $\mathbf{p 1 0}$ & $\mathbf{p 5 0}$ & $\mathbf{p 9 0}$ \\
\hline 24 & 1,79 & 2,15 & 2,54 \\
\hline 25 & 1,83 & 2,19 & 2,57 \\
\hline 26 & 1,87 & 2,22 & 2,59 \\
\hline 27 & 1,91 & 2,26 & 2,62 \\
\hline 28 & 1,95 & 2,29 & 2,65 \\
\hline 29 & 1,99 & 2,33 & 2,68 \\
\hline 30 & 2,04 & 2,36 & 2,71 \\
\hline 31 & 2,08 & 2,40 & 2,74 \\
\hline 32 & 2,12 & 2,43 & 2,77 \\
\hline 33 & 2,16 & 2,47 & 2,80 \\
\hline 34 & 2,20 & 2,50 & 2,83 \\
\hline 35 & 2,25 & 2,54 & 2,86 \\
\hline 36 & 2,29 & 2,57 & 2,89 \\
\hline 37 & 2,33 & 2,61 & 2,92 \\
\hline 38 & 2,37 & 2,64 & 2,95 \\
\hline 39 & 2,41 & 2,68 & 2,98 \\
\hline 40 & 2,45 & 2,71 & 3,01 \\
\hline 41 & 2,50 & 2,75 & 3,04 \\
\hline 42 & 2,54 & 2,78 & 3,07 \\
\hline
\end{tabular}

Información conjunta Alarcón y Pittaluga. 


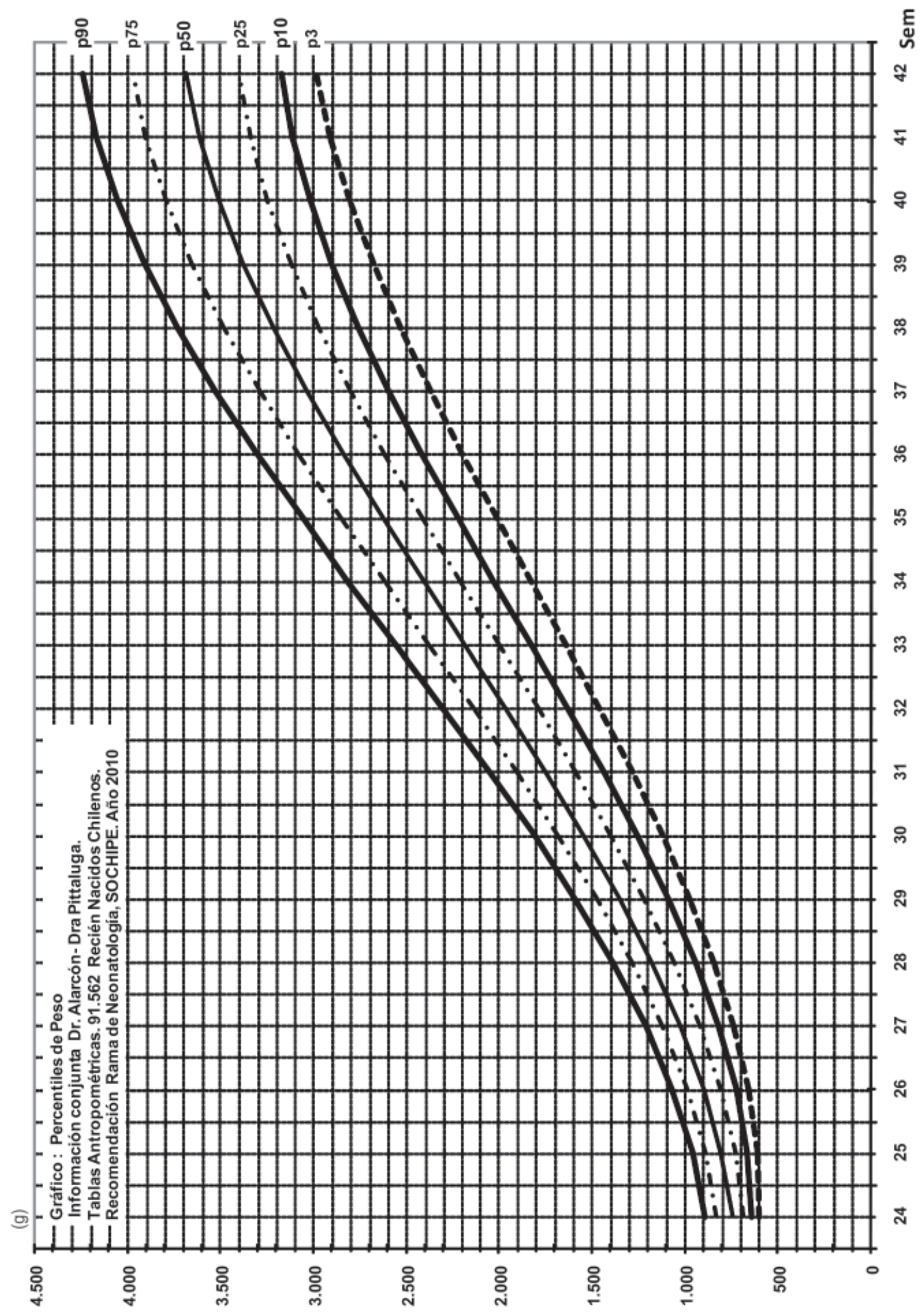

Figura 1. Percentiles de peso según EG. 


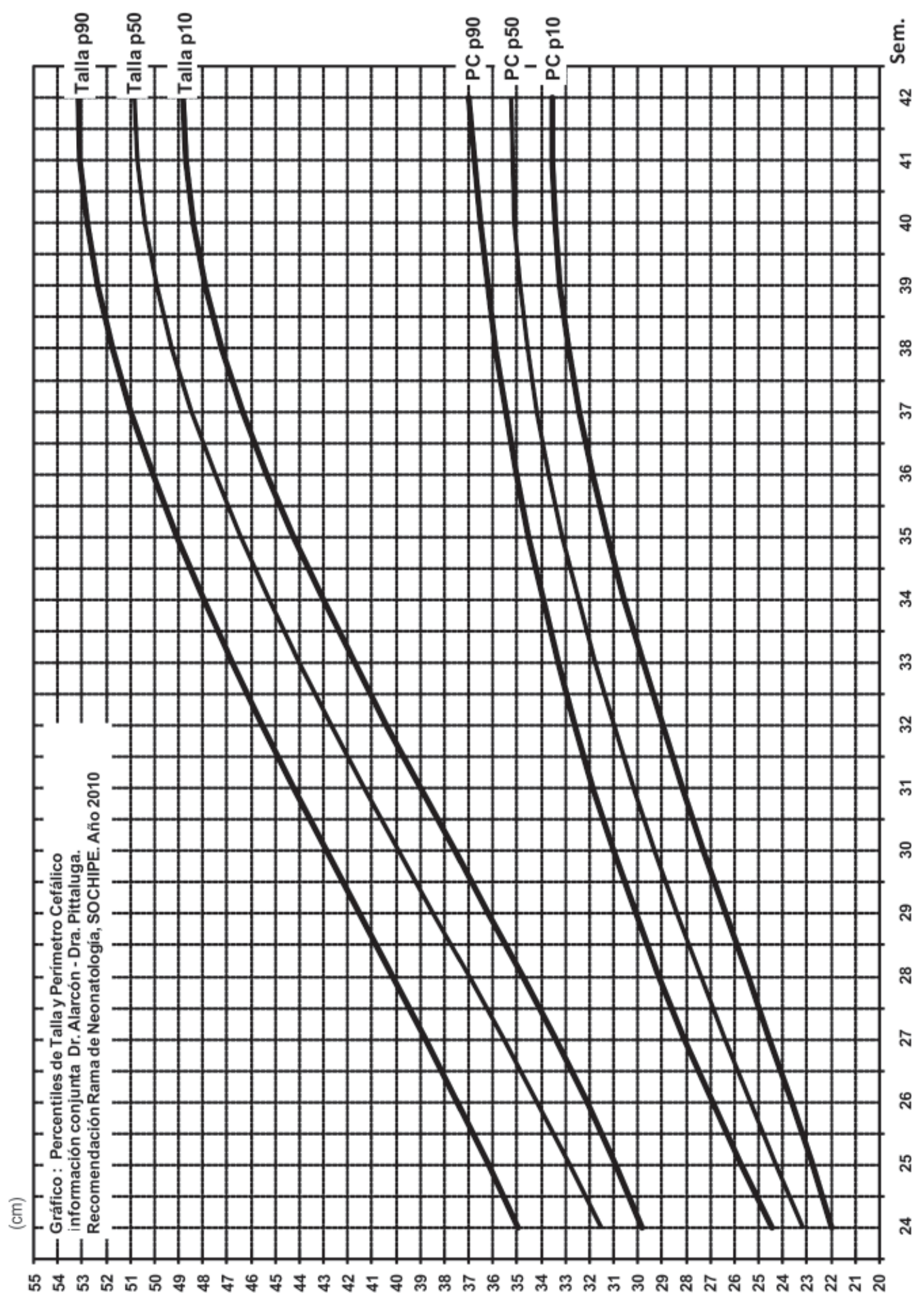

Figura 2. Percentiles de Talla y Perímetro Craneano según EG. 
M. MILAD A. y cols.

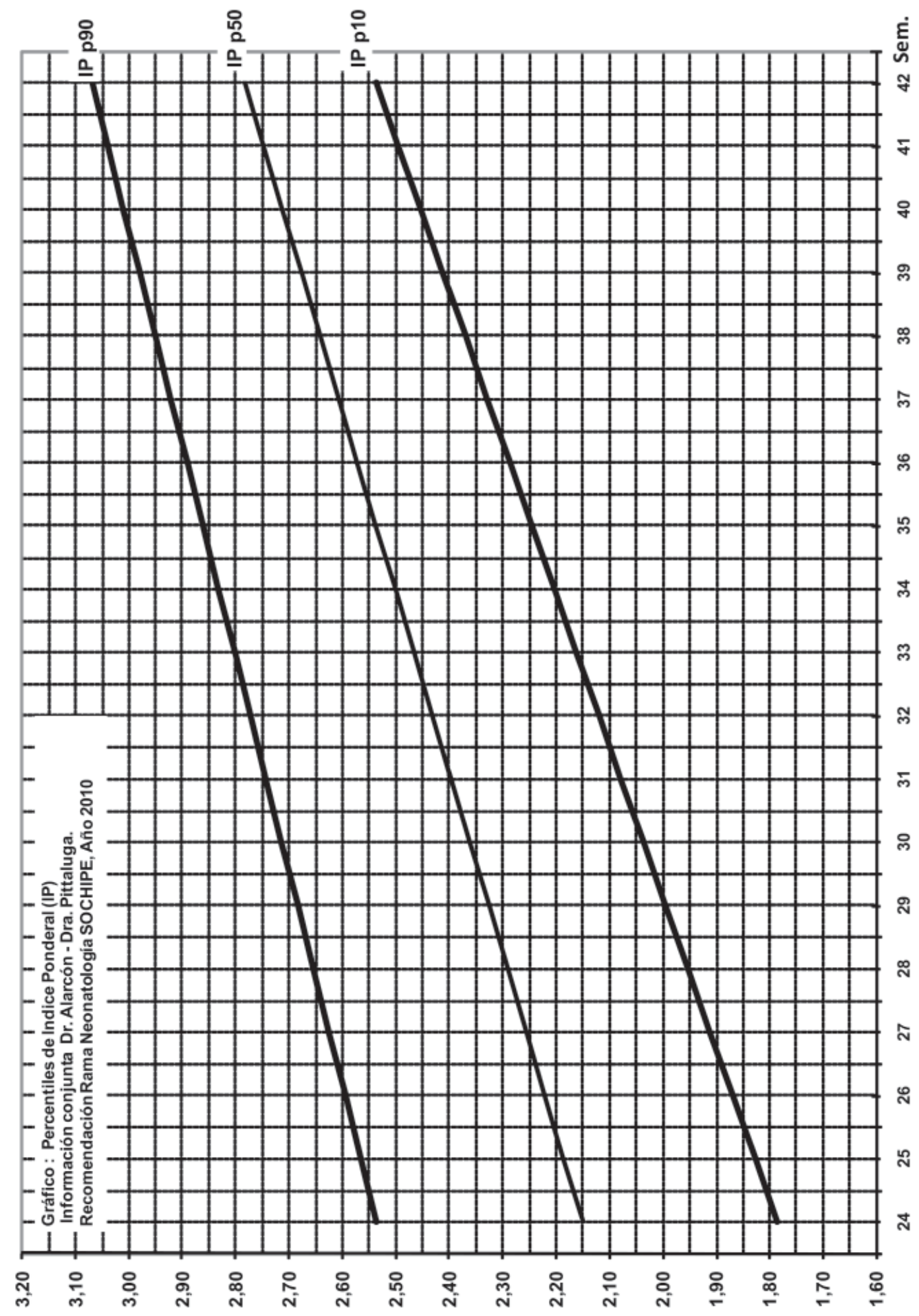

Figura 3. Percentiles de Índice Ponderal (peso (g) x 100 / talla $^{3}(\mathrm{~cm})$ ) según EG. 


\section{Agradecimiento}

Agradecemos la gran generosidad de los Dres. Pittaluga y Alarcón para facilitar los datos matrices de sus estudios, lo cual permitió confeccionar estas nuevas tablas y curvas en las que se basa esta recomendación y en especial reconocemos el fundamental el aporte del Dr. Alarcón en consolidar la información de las matrices.

\section{Referencias}

1.- De Onis M, Habicht JP: Anthropometric reference data for international use: recommendations from a World Health Organization Expert Committee. Am J Clin Nutr 1996; 64: 650-8

2.- Garner P, Panpanich R, Logan S: Is routine Growth monitoring effective? A systematic review of trials. Arch Dis Child 2000; 82: 197-201.

3.- Clausson B, Gardosi J, Francis A, Cnattingius S: Perinatal outcome in SGA births defined by customized versus population-based birth weight standards. BJOG 2001; 108: 830-4.

4.- McIntire DD, Bloom SL, Casey BM, Leveno KJ: Birth weight in relation to morbidity and mortality among newborn infants. N Engl J Med 1999; 340: 1234-8.

5.- Garite TJ, Clark R, Thorp JA: Intrauterine growth restriction increases morbidity and mortality among premature neonates. Am J Obstet Gynecol 2004; 191: 481-7.

6.- Latal-Hajnal B, von Siebenthal K, Kovari H, et al: Postnatal growth in VLBW infants: significant association with neurodevelopmental outcome. J Pediatr 2003; 143 (2): 163-70

7.- Law CM, Shiell AW, Newsome CA, et al: Fetal, infant, and childhood growth and adult blood pressure: a longitudinal study from birth to 22 years of age. Circulation 2002; 105 (9): 1088-92.

8.- Shripara CR., Jeffrey T: Growth curves for preterm infants. Early Human Development 2007; 83: 643-51.

9.- WHO Working Group. Use and interpretation of anthropometric indicators of nutritional status. Bull World Health Organ 1986; 64: 929-41.

10.- WHO Multicenter Growth Reference Study Group: WHO Child Growth Standards. Acta Pediatr Suppl 2006; 450: 5-101.

11.- Juez G, Ventura-Juncá P, Lucero E: Crecimiento intrauterino en un grupo seleccionado de $\mathrm{RN}$ chilenos.
Subdiagnóstico de Retardo de Crecimiento Intrauterino en Chile. Rev Méd Chile 1984; 112: 759-64.

12.- Pittaluga PE, Díaz AV, Mena NP, et al: Curva de crecimiento intrauterino para prematuros entre 23 a 36 semanas de edad gestacional. Rev Chil Pediatr 2002; 73 (2): 135-41 ISSN 0370-4106.

13.- González RP, Gómez RM, Castro RS, et al: Curva nacional de distribución de peso al nacer según edad gestacional: Chile, 1993 a 2000. Rev Med Chile 2004; 132: 1155-65

14.- Alarcón J, Alarcón Y, Hering E, Buccioni R: Curvas antropométricas de RN chilenos. Rev Chil Pediatr 2008; 79 (4): 364-372.

15.- Carvajal JA, Vera C, Vargas P, Oyarzún E: Subdiagnóstico de restricción de crecimiento fetal mediante la aplicación de las curvas de crecimiento intrauterino del Ministerio de Salud. Rev Med Chile 2007; 135: 436-42.

16.- Pittaluga PE, Mena P, Díaz V, Gederlini A: Curvas de peso neonatal para la identificación de una población de riesgo. Libro de Resúmenes II Congreso Chileno de Neonatología 19-20 Octubre 2006.

17.- Zaw W, Gagnon R, da Silva $O$ : The risks of adverse neonatal outcome among preterm small for gestational age infants according to neonatal versus fetal growth standards. Pediatrics 2003; 111: 1273-7.

18.- Marconi AM, Ronzoni S, Battaglia FC: Comparison of Fetal and Neonatal Growth Curves in Detecting Growth Restriction. Obstet Gynecol 2008; 112: 1227-34.

19.- Ben-Haroush A, Yogev Y, Bar J, et al: Accuracy of sonographically estimated fetal weight in 840 women with different pregnancy complications prior to induction of labor. Ultrasound Obstet Gynecol 2004; 23: 172-6.

20.- Ott $W$ : Intrauterine growth retardation and preterm delivery. Am J Obstet Gyneco 1993; 168: 1710-7.

21.- Bukowsky R, Gahn D, Denning J, Saade G: Impairment of growth in fetuses destined to deliver preterms. Am J Obstet Gynecol 2001; 185: 463-7.

22.- Royal College of Obstetricians and Gynaecologists (RCOG): The investigation and management of the small for gestational age fetus. RCOG. Green Top guideline $\mathrm{N}^{\circ} 31$ London. UK.

23.- Lubchenco LO, Hansman C, Dressler M, Boyd E: Intrauterine growth as estimated from live born birth weight data at 24 to 42 weeks of gestation. Pediatrics 1963; 32: 793-800.

24.- Nutrtional Committee Canadian Paediatrics Society: Use of growth charts for assessing and monitoring growth in Canadian infants and children. Paediatr Child Health 2004; 9: 171-80.

25.- Karna P, Brooks K, Muttineni J, Karmaus W: Anthro- 
M. MILAD A. y cols.

pometric measurements for neonatos, 23 to 29 weeks gestation, in the 1990s. Paediatr Perinat Epidemiol 2005; 19: 215-26.

26.- Fenton TR: A new growth chart for preterm babies: Babson and Benda's chart updated with recent data and a new format. BMC Pediatr 2003; 16 (3): 13.
27.- Bertino E, Milani S, Fabris C, De Curtis M: Neonatal anthropometric charts: what they are, what they are not. Arch Dis Child Fetal Neonatal Ed 2007; 92: F7-F10.

28.- Olsen IE, Groveman SA, Lawson L, Clark RH, Zemel $B S$ : New Intrauterine Growth Curves Based on United States Data. Pediatrics 2010; 125: e214-24. 